\title{
Analysis of the Nuclear Fusion Process Based on Potential Barriers Modified by Coupling Effects
}

\author{
H.D. Marta ${ }^{1}$, M.R. Spinella ${ }^{2,3}$, J.E. Testoni ${ }^{2,3}$, and O. Dragún ${ }^{2}$ \\ ${ }^{1}$ Instituto de Física, Facultad de Ingeniería, C.C. 30, C.P. 11000, Montevideo, Uruguay \\ ${ }^{2}$ Departamento de Física, Comisión Nacional de Energía Atómica, \\ Avda. del Libertador 8250, 1429 Buenos Aires, Argentina \\ ${ }^{3}$ Also at CONICET, Argentina
}

Received on 22 September, 2003

\begin{abstract}
Partial wave analysis and the decoupling of the wave equations based on potential barriers modified by coupling effects are applied in the study of the fusion process in medium heavy nuclei. Several relevant physical quantities are calculated in order to provide evidence of underline reaction mechanisms that determine characteristics of fusion excitation functions and barrier distributions. The influence of $\mathrm{Q}-$ values is especially considered. The method is applied for the neutron transfer reaction in the ${ }^{17} \mathrm{O}+{ }^{144} \mathrm{Sm}$ system.
\end{abstract}

\section{Introduction}

Open reaction channels in nuclear collisions leading to fusion processes induce structure dependent effects in the fusion excitation functions [1]. The concept of barrier distributions, expressed as the second derivative respect to the incident energy of the excitation functions times this energy, is an useful tool in order to put in evidence these structural effects [2].

In Ref. [3] we have recently proposed the energydependent modified potential barriers that include coupling effects as an appropriate method for providing an insight into the reaction mechanisms. It is our purpose to extend the study concerning the inelastic excitation of the $1.81 \mathrm{MeV}$ $3^{-}$vibrational state of the target nucleus in the ${ }^{16} \mathrm{O}+{ }^{144} \mathrm{Sm}$ system, performed in that work, to one-neutron transfer reactions in the ${ }^{17} \mathrm{O}+{ }^{144} \mathrm{Sm}$ system. Experimental data in which the influence of the one-neutron pick-up on the fusion barrier distribution is apparent are available [4].

\section{The formalism}

The basic coupled-channel formalism used in the present work has been addressed in detail in Ref. [3]. The radial wavefunctions $g_{n \ell}(r)$ which describe the collision process are found by solving the following set of coupled equations,

$$
\begin{array}{r}
{\left[\frac { \hbar ^ { 2 } } { 2 \mu _ { n } } \left(\frac{d^{2}}{d r^{2}}-\frac{\ell(\ell+1)}{r^{2}}\right.\right.} \\
\left.\left.+k_{n}^{2}\right)-V_{C}(r)\right] g_{n \ell}(r) \\
=\sum_{n^{\prime}} V_{n, n^{\prime}}(r) g_{n^{\prime} \ell}(r) .
\end{array}
$$

The diagonal matrix elements $V_{n, n}(r)$, for both, the elastic and the transfer channels, are represented by the same conventional Woods-Saxon shaped nuclear optical potential. The symmetrical nondiagonal formfactors that link the elastic and transfer channels are given by the expression

$$
V_{n, n^{\prime}}(r)=F \exp \left(-\left(r-R_{t r}\right) / a_{t r}\right)
$$

In order to analyze the behavior of the fusion barrier distributions, energy-dependent potential barriers modified by coupling effects given by the expression

$$
\begin{aligned}
V_{n \ell}^{\text {mod }}(r)= & \frac{\hbar^{2}}{2 \mu_{n}} \frac{\ell(\ell+1)}{r^{2}}+V_{C}(r) \\
& +\frac{\sum_{n^{\prime}} V_{n, n^{\prime}}(r) g_{n^{\prime} \ell}(r)}{g_{n \ell}(r)},
\end{aligned}
$$

were proposed in Refs. [3, 5] .

Several physical quantities will be addressed, i.e. fusion excitation functions, $\sigma^{f}(E)$; fusion barrier distributions, $B(E)$; sources (or sinks), $S(r)$; and incoming and outgoing currents, $C(r)$; (see Ref. [3]).

\section{Analysis of fusion cross sections and barrier distributions}

\subsection{The influence of the channel coupling}

The fusion excitation functions, $\sigma^{f}(E)$, and the corresponding barrier distributions, given by the second derivative of the fusion cross section times the incident energy respect to this energy,

$$
B(E)=d^{2}\left(E \sigma^{f}\right) / d E^{2},
$$


are shown in Fig. 1 for the neutron stripping $(Q=2.6 \mathrm{MeV})$ and the neutron pick-up $(Q=-2.48 \mathrm{MeV})$ in the ${ }^{17} \mathrm{O}+{ }^{144} \mathrm{Sm}$ system.
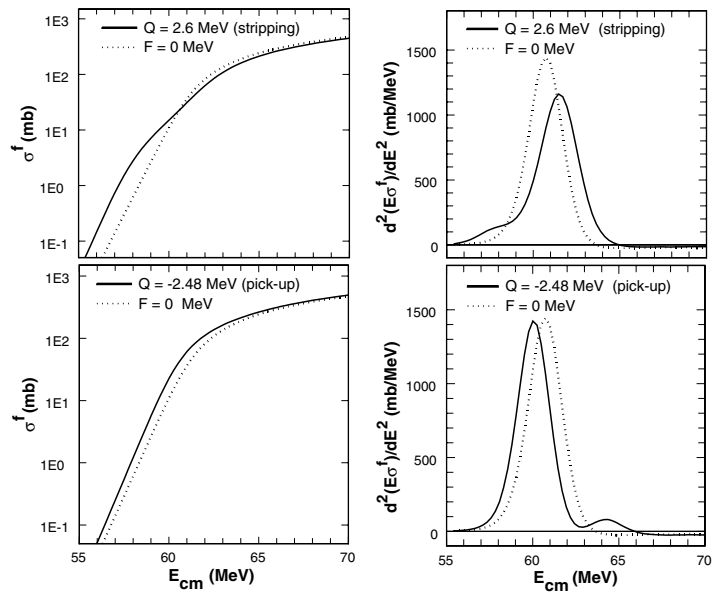

Figure 1. Fusion excitation functions and the corresponding barrier distributions when a neutron stripping or a neutron pick-up channel is open in the ${ }^{17} \mathrm{O}+{ }^{144} \mathrm{Sm}$ system.

As a reference, $\sigma^{f}(E)$ and $B(E)$, for the case in which the transfer channel is closed, i.e. $F=0 \mathrm{MeV}$, are plotted. Sub-Coulomb cross sections show an enhancement when the transfer channel is open. A moderate hindrance in the cross sections at energies over the Coulomb barrier takes place for the positive $Q$-value. (In the following, $Q$-values are arbitrary selected just for illustrative reasons.) The barrier distributions reveal a splitting of the single peak corresponding to $F=0 \mathrm{MeV}$ as consequence of the inclusion of an open transfer channel with $F=1.5 \mathrm{MeV}$; the split peaks are, in these cases, of different magnitude. The peak at the left in the plot for positive $Q$ is quite smaller than the peak that arises at the right. The situation is reversed in the case of negative $Q$. In both instances the reference peak is placed between the split peaks.

One might try to associate each split peak with one of the two involved channels, assigning, for instance, the large peaks to the elastic channel and the small ones to the transfer channel. Fig. 2 illustrates this issue; Fig. 2a and Fig. 2c represent the contribution of each channel to the fusion cross section. The fusion through the transfer channel dominates in the case of positive $Q$ and through the elastic channel for negative $Q$. These partial excitation functions originate two components in the barrier distributions, related to each reaction channel, as shown in Fig. 2b and Fig. 2d; their addition constitutes the total fusion barrier distributions. It is apparent that the assignation of the clearly separated peaks in the total distribution to the individual reaction channels is not a simple task. In fact, the small peak in the positive $Q$ case has a neat contribution from the transfer channel. However, that corresponding for negative $Q$ is determined by the balance of relatively small differences between the positive and negative contributions of the elastic and transfer channels. The big peaks, in both cases, are built by important contributions of both channels. Therefore, the univocal assignation of a channel to a particular peak is not, in general, obvious.
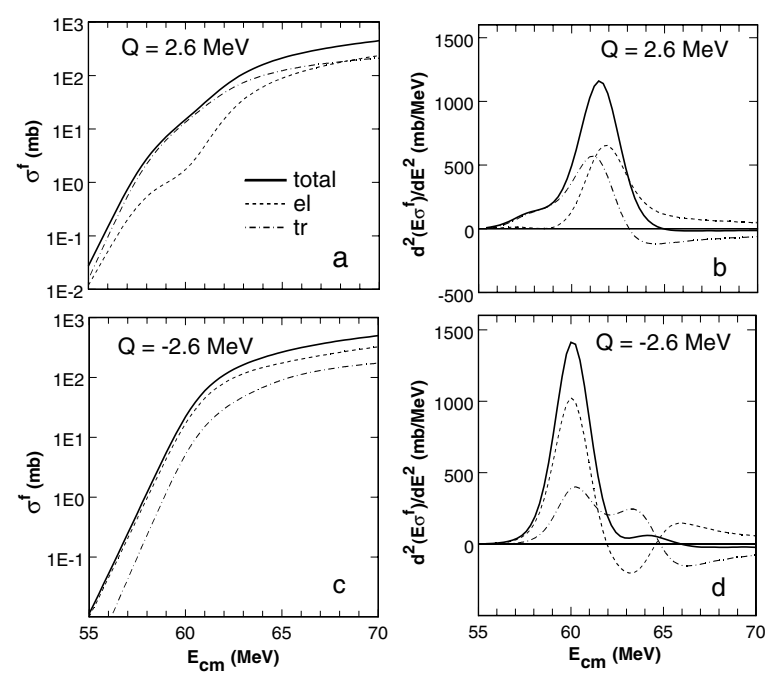

Figure 2. Contributions of the elastic and the transfer channels to the total fusion excitation function and barrier distributions for positive and negative $Q$-values.

\subsection{Partial waves analysis}

In order to understand some features of the behavior of fusion cross sections and barrier distributions, it is useful to take into account that we deal with central forces. Consequently, the different analyses can be performed on the basis of independent sets of wavefunctions characterized by a relative angular momentum $\ell \hbar$. In this way, a barrier distribution can be developed in partial barriers associated to each $\ell$-value.

Figure 3a shows how the fusion barrier distribution, for the simple case without coupling, i.e. $F=0 \mathrm{MeV}$, is built-up as a sum of partial barriers that interfere among them; they have oscillating shapes presenting a positive peak followed by a negative one which asymptotically decays to zero. The plot showing all the partial barriers permits to visualize a characteristic envelope. In the example, up to the top of the peak, the contributions for different angular momenta furnish only positive values, given place to the rise of the characteristic peak. Then, the presence of negative values produces the descendent side of the peak. For higher energies, in spite of being each partial barrier progressively bigger, they thoroughly cancel among them when they are added.

If the transfer channel is open, a second structure can be visualized in the plot of the partial barriers as a distinct new envelope as shown by Fig. 3b. This fact is due to the presence of a second oscillation in the individual partial barriers that, when they superimpose, give place to an additional peak in the total barrier distribution. 

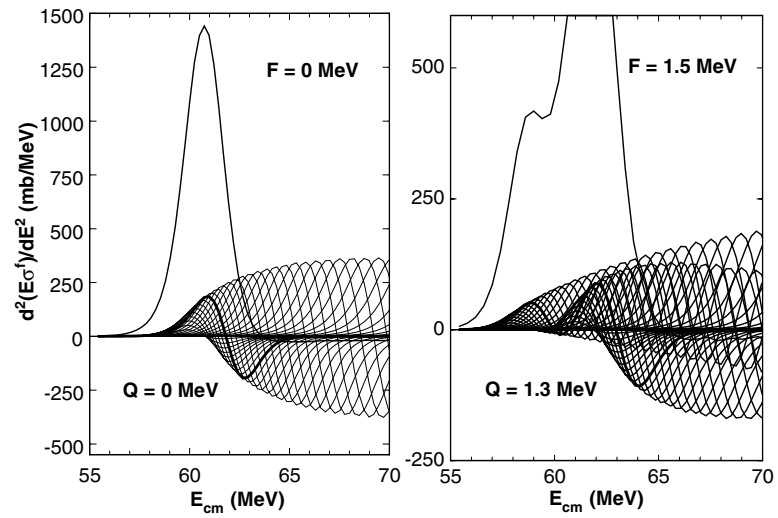

Figure 3. Decomposition of the fusion barrier distribution for cases without coupling (a) and with coupling (b) according to the contributions of the different relative angular momenta $\ell \hbar$. The partial barrier distributions for $\ell=9$ are highlighted.

Particular features of the reaction mechanisms present in the fusion process can be studied considering their incidence on the partial fusion barrier distribution for each $\ell$-value. As illustrates the highlighted partial barrier in the previous figures, the angular momentum $\ell=9$ might be a good choice in order to study the behavior of a representative partial barrier distribution for the present case.

\subsection{Potential barriers modified by coupling}

In the upper-row of Fig. 4, the total excitation functions for stripping transfer for different $Q$-values, together with the excitation functions for $F=0 \mathrm{MeV}$, i.e. when the transfer channel is closed, are shown. In the lower-row, the corresponding partial contributions for $\ell=9$ are plotted.
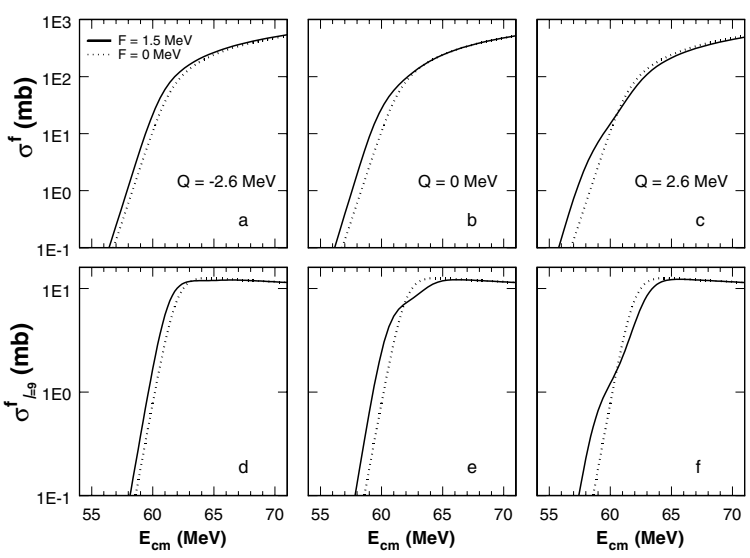

Figure 4. Total and partial $(\ell=9)$ excitation functions for different Q-values.

Figure 5 show the potential barriers modified by coupling effects, $V_{n \ell}^{\bmod }(r)$, for selected energies, in order to illustrate some paradigmatic situations (see Eq. (3)). As shown in Fig. 4a, an enhancement of the cross sections for stripping transfer with $Q=-2.6 \mathrm{MeV}$ can be observed around the Coulomb barrier energy, $E_{C}=61.65 \mathrm{MeV}$, when it is compared with the $F=0 \mathrm{MeV}$ case. This fact is corroborated in Fig. 5a, where the conventional barrier and the elastic modified barrier for $E_{c m}=61.4 \mathrm{MeV}$ are plotted. At such energy, the conventional barrier is over the modified barrier, and the elastic channel energy is in between them. From the point of view of the conventional barrier we are dealing with a sub-barrier situation, while it is not the case from the point of view of the modified barrier. Consequently, it follows that an enhancement of the fusion cross section is to be expected.
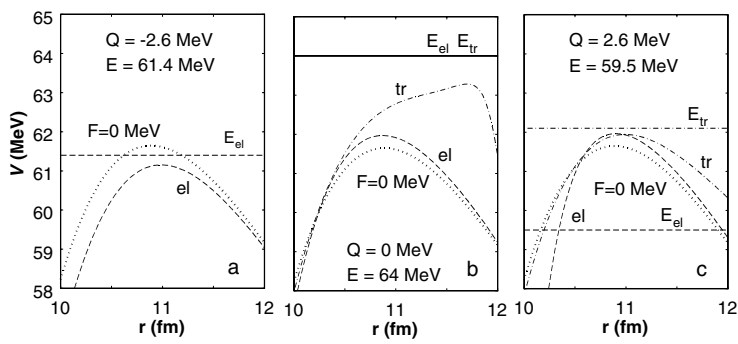

Figure 5. Modified barriers compared with the conventional barrier for different $Q$-values and $\ell=9$.

In the case of $Q=0 \mathrm{MeV}$, the Fig. $4 \mathrm{~b}$ indicates hindrance over the Coulomb barrier and enhancement below it. At $64 \mathrm{MeV}$, the elastic, as well as the transfer modified barriers, are over the conventional one as can be seen in Fig. 5b; this situation means hindrance. At energies below $E_{C}$ a situation alike to that described for the negative $Q$-value takes place.

As shown by Fig $4 \mathrm{c}$, for $Q=2.6 \mathrm{MeV}$, a hindrance similar to that mentioned in the case of $Q=0 \mathrm{MeV}$ occurs in the partial excitation function for energies greater than $61 \mathrm{MeV}$, and at lower energies an enhancement is present. As can be seen in Fig. 5c at $59.5 \mathrm{MeV}$ the fact that the modified barriers are over the conventional barrier would suggest hindrance and not enhancement. This apparent contradiction is clarified when it is observed that the energy in the transfer channel surpasses the transfer modified barrier top, while that in the elastic one is well below the top of the conventional barrier that is responsible for the magnitude of the cross section in the $F=0 \mathrm{MeV}$ reference case. Consequently, the fusion through the transfer channel is predominant, and an enhancement takes place.

\subsection{The interplay between relevant physical quantities}

Figure 6 addresses the interplay between sources, $S$, currents, $C$, and barriers modified by coupling effects, $V^{\text {mod }}$, for different illustrative cases. (Dashed lines refers to the elastic channel and dot-dashed lines to the transfer channel.) The modified barriers are plotted in the cases of $Q=-2.6 \mathrm{MeV}$ for $E_{c m}=59 \mathrm{MeV}$, and $Q=2.6 \mathrm{MeV}$ for $E_{c m}=60 \mathrm{MeV}$. The figures in the low row represent the 
sources and the incoming (negative) and outgoing (positive) currents in the channels. For negative $Q$-value, the source is placed outside the transfer modified barrier; the channel energies are under the top of the barriers for both channels.
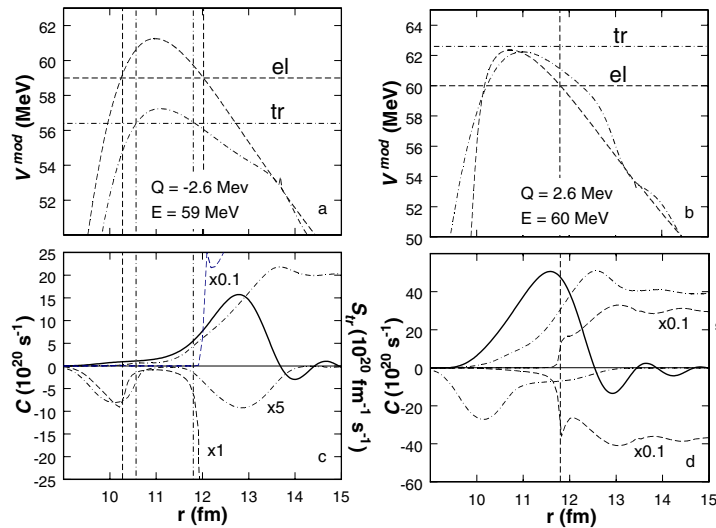

Figure 6. Interplay between sources, $S$ (full line), currents, $C$, and modified barriers, $V_{n \ell}^{\text {mod }}(r)$, at sub-Coulomb energies for $\ell=9$ (see text).

Most of the current is reflected and contributes to the elastic scattering and the transfer reaction. A small current, however, goes into the barrier and scarcely contributes to fusion. Dips in the incoming currents between the turning points of the specific modified barrier can be observed. It can be verified how, in the case of positive $Q$, at low energies, the fusion process essentially comes about through the transfer channel; the energy for the transfer channel is over the top of the corresponding modified barrier, while that for the elastic channel is under the top of its barrier. The position of the source is, in the present case, quite coincident with that of the barrier, but placed somewhat in the external region. The incoming elastic current is thoroughly reflected and minimally contributes to fusion.

\subsection{Summary}

We summarize some characteristics of our approach to the study of the nuclear fusion in medium-heavy nuclei collisions, illustrated through the analysis of the ${ }^{17} \mathrm{O}+{ }^{144} \mathrm{Sm}$ system with a neutron transfer open channel. The influence of the Q-values is specially considered: a) The numerical calculation of wavefunctions describing the system in two channels - the elastic one and that corresponding to the transfer of one neutroncoupled by the mutual interaction in the collision of the nuclei.

b) The introduction of a third channel, the fusion process, by considering that all the absorption described by the optical model potential leads to fusion.

c) The decomposition in partial waves that allows us the evaluation of several physical quantities —as a function of the distance between nuclei or incident energy - for particular representative relative angular momenta, i.e. potential barriers modified by coupling effects, probability densities, fusion excitation functions, the fusion barrier distributions, fusion rates, currents, and sources. These quantities permit the study of different aspects of the fusion process. Such analyses are not possible considering the total cross sections and barrier distributions.

d) The introduction of the formal decoupling through the potentials modified by coupling effects that allows us a simple comprehension of different features of the underlying reaction mechanism.

\section{References}

[1] W. Reisdorf, J. Phys. G: Nucl. Part. Phys. 20, 1297 (1994) and referencies therein.

[2] N. Rowley, G.R. Satchler, and P.H. Stelson, Phys. Lett. B254, 25 (1991); M. Dasgupta, D.J. Hinde, N. Rowley, and A.W. Stefanini, Annu. Rev. Nucl. Part. SCI 48, 401 (1998).

[3] M.R. Spinella, J.E. Testoni, O. Dragún, and H.D. Marta, Nucl. Phys. A687, 4385 (2001)

[4] C.R. Morton, M. Dasgupta, D.J. Hinde, J.R. Leigh, R.C. Lemmon, J.P. Lestone, J.C. Mein, J.O. Newton, H. Timmers, N. Rowley, and A.T. Kruppa, Phys. Rev. Lett. 72, 4074 (1994); J.R. Leigh, M. Dasgupta, D.J. Hinde, J.C. Mein, C.R. Morton, R.C. Lemmon, J.P. Lestone, J.O. Newton, H. Timmers, J.X. Wei, and N. Rowley, Phys. Rev. C52, 3151 (1995).

[5] J.E. Testoni, O. Dragún, H. Massmann, and M.R. Spinella, Nucl. Phys. A669, 173 (2000). 